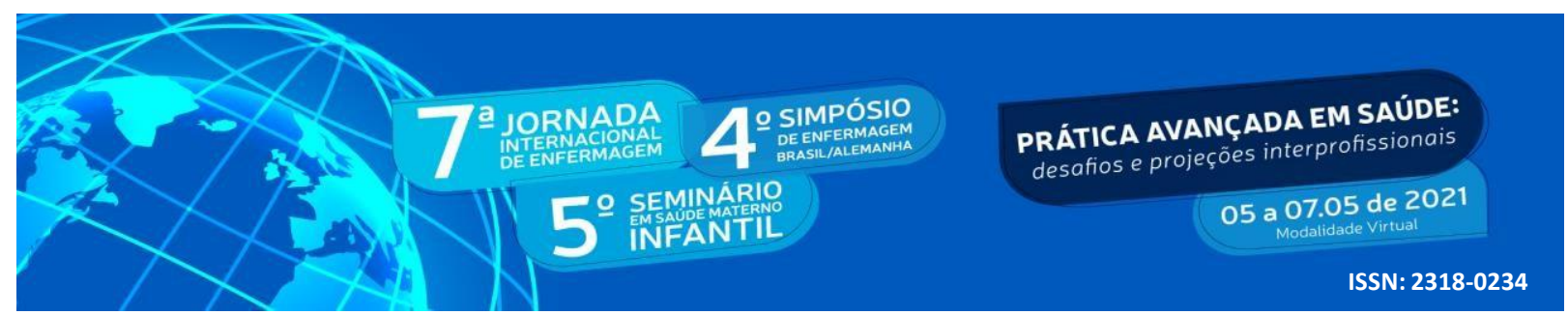

DOI: http://doi.org/10.48195/jie2021-034

\title{
GRUPO DE GESTANTES NA ATENÇÃO PRIMÁRIA EM SAÚDE: PRÁTICA EDUCATIVA BASEADA EM METODOLOGIA TEAM-BASED LEARNING ${ }^{1}$
}

\author{
Joselaine Rigue ${ }^{2}$; Maclaine de Oliveira Roos ${ }^{3}$; Marcia Dal Bem Cherobini' ${ }^{4}$; \\ ClaudiaZamberlan 5
}

\begin{abstract}
RESUMO
A mulher vivencia durante o período gravídico-puerperal diferentes sentimentos dentre eles as angústias, incertezas e dúvidas inerentes a este período tão singular em suas vidas. Ao refletir esse contexto, objetiva-se descrever o planejamento de uma atividade online de educação em saúde utilizando o Team-Based Learning (TBL) para um grupo de gestantes na atenção primária em saúde. Configura-se como uma nota prévia de um material elaborado por três mestrandas do Mestrado Profissional em Saúde Materno Infantil da Universidade Franciscana (UFN), como proposta da disciplina de Educação em Saúde. Para tal, elegeu-se a metodologia Team-Based Learning (TBL) para operacionalizar as atividades de educação em saúde. Espera-se que esta atividade por meio da utilização de uma metodologia ativa, promova discussões e reflexões que auxiliem na compreensão das gestantes em relação ao parto.
\end{abstract}

Palavras-chave: Educação em saúde; Gestante; Metodologia ativa.

\begin{abstract}
GROUP OF PREGNANT WOMEN IN PRIMARY HEALTH CARE: EDUCATIVE PRACTICE FUNDAMENTED ON TEAM-BASED LEARNING METHODOLOGY
\end{abstract}

During the pregnancy-puerperal period, women experience different feelings, including the anxieties, uncertainties and doubts inherent in this very unique period in their lives. Reflecting this context, the objective is to describe the planning of an online health education activity using Team-Based Learning (TBL) for a group of pregnant women in primary health care. Such description is configured as a preliminary note of a material prepared by three master's students of the Professional Master's in Maternal and Child Health at the Universidade Franciscana (UFN) [Franciscan University], as a

\footnotetext{
1 Nota prévia

${ }^{2}$ Enfermeira. Mestranda do Mestrado profissional em saúde Materno Infantil. Universidade Franciscana-UFN.

E-mail: josirigue01@gmail.com

${ }^{3}$ Pediatra. Mestranda do Mestrado Profissional em Saúde Materno Infantil. UFN. E-mail:

maclaine@terra.com.br

${ }^{4}$ Enfermeira. Mestranda do Mestrado Profissional em Saúde Materno Infantil. UFN. E-mail: marcinhacherobini@gmail.com

5 Enfermeira. Docente do Mestrado profissional em Saúde Materno Infantil. UFN. E-mail: czamberlan23@gmail.com
} 


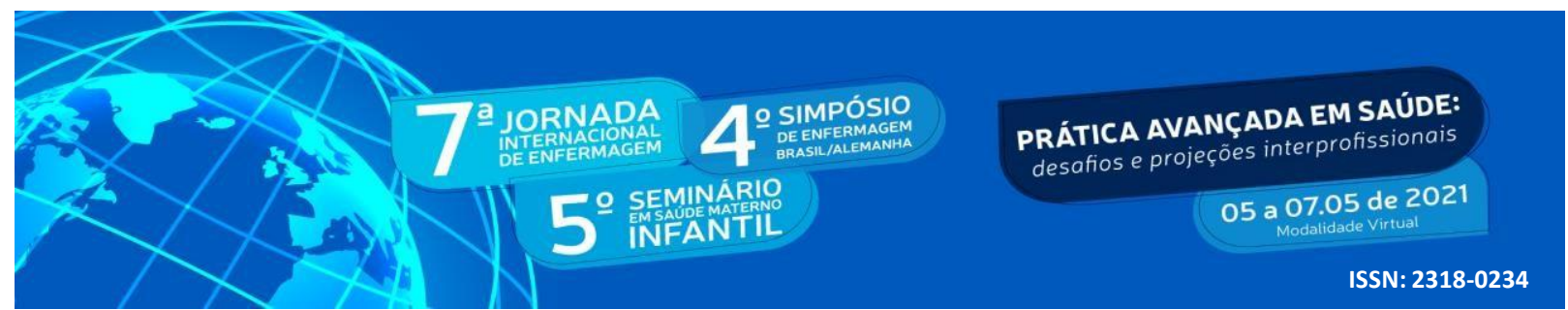

proposal of the discipline of Health Education. To this end, the Team-Based Learning (TBL) methodology was chosen to operationalize health education activities. Through the use of an active methodology, it is expected that this activity promotes discussions and reflections that assist in the understanding of pregnant women in relation to childbirth.

Keywords: Health education; Pregnant; Active methodology.

\section{INTRODUÇÃO}

A gestação é um período especial e muito singular na vida da mulher, permeado de diferentes significados e valores. As mudanças e adaptações necessárias nessa fase, muitas vezes culminam com diferentes sentimentos dentre eles o medo, a insegurança e a incerteza (PEREIRA et al.,2017). Diante desses pressupostos, faz-se necessário que os profissionais de saúde proporcionem uma assistência qualificada e humanizada que contemple as necessidades das gestantes assistidas (SILVA et al., 2016).

Para tal, acredita-se que o desenvolvimento de práticas de educação em saúde mediadas por metodologias ativas são relevantes, uma vez que auxiliam no empoderamento tanto individual quanto coletivo, corroborando com a autonomia das gestantes em relação ao ciclo gravídico-puerperal (GURGEL, 2015).

Portanto, esta temática corrobora com o alcance dos Objetivos de Desenvolvimento do Sustentável (ODS), destacando-se a ODS 3.1 que visa até 2030, reduzir a taxa de mortalidade materna global para menos de 70 mortes por 100.000 nascidos vivos e a ODS 3.2, que visa acabar com as mortes evitáveis de recém-nascidos e crianças menores de 5 anos em todos os países, objetivando reduzir a mortalidade neonatal (ONU, 2015). Nesse sentido, elegeu-se a TBL como ferramenta educativa para viabilizar um grupo de gestantes para uma prática educativa online visto que a pandemia da COVID-19 inviabiliza, neste momento, a realização de atividades grupais desenvolvidas anteriormente na Unidade Básica de Saúde (UBS). Entende-se que os profissionais de saúde devam reinventar-se nas suas práticas de trabalho transcendendo as práticas tradicionais a fim de viabilizar o alcance das demandas de saúde da sua comunidade.

\section{OBJETIVO}




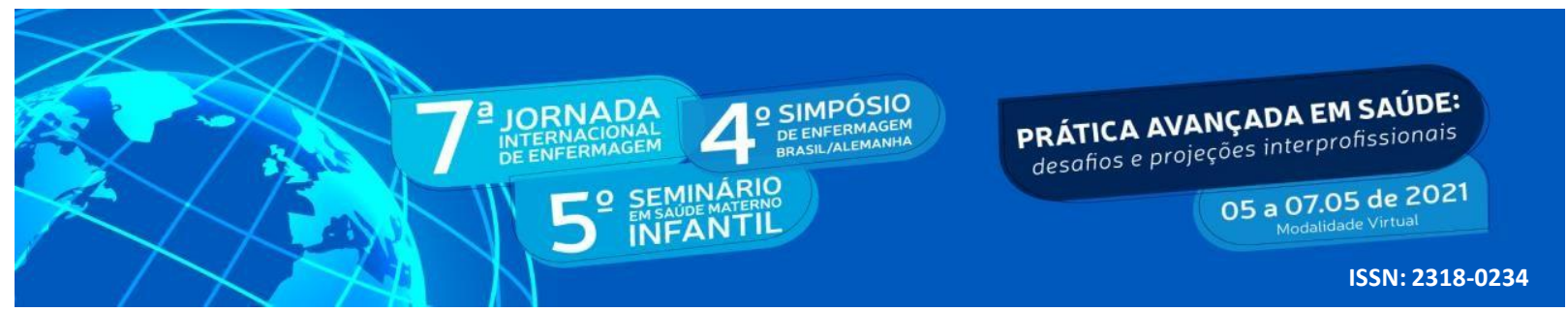

Descrever o planejamento de uma atividade online de educação em saúde utilizando o Team-Based Learning (TBL) para um grupo de gestantes na atenção primária em saúde.

\section{METODOLOGIA}

Trata-se de uma nota prévia proposta como atividade da disciplina de Educação em Saúde do Mestrado Profissional em Saúde Materno Infantil da Universidade Franciscana (UFN), no município de Santa Maria, RS, o qual foi desenvolvido por três mestrandas no ano de 2020 sob orientação da docente da disciplina. A atividade será desenvolvida no decorrer do mês de abril de 2021, e será dividida em 3 momentos, com duração de aproximadamente 50 minutos para cada encontro. A plataforma digital para a realização dos grupos junto às gestantes será através do Google meet. A metodologia foi planejada para ser aplicada online, devido ao momento atual de pandemia por Covid 19, porém posteriormente poderá ser aplicada de forma presencial e também disponibilizada para outros serviços de saúde do município.

Para operacionalizar as atividades educativas grupais a metodologia utilizada será a TBL. O TBL é uma metodologia ativa que proporciona um ambiente motivador e cooperativo, pois permite a construção do conhecimento de forma dinâmica, crítica, reflexiva e interativa, possibilitando que os participantes sejam protagonistas de seu aprendizado (FARIAS; MARTINS; CRISTO, 2015).

Referente à organização da atividade seguiram-se os quatro passos propostos como referencial metodológico para sustentar cientificamente a utilização do TBL, considerando as quatro etapas necessárias que caracterizam esse método conforme destacam as evidências científicas que retratam esse método ativo de aprendizagem (OLIVEIRA et al., 2018; FARIAS; MARTIN; CRISTO, 2015).

\section{RESULTADOS PRÉVIOS}

Uma das facilitadoras, que é vinculada a uma equipe de UBS de um município do interior do RS, realizou a busca por gestantes que apresentavam uma idade gestacional de aproximadamente 22 semanas e de diferentes faixas etárias. Essas gestantes foram contatadas durante as consultas na UBS e também via telefone, totalizando 14 gestantes. Objetivou-se 


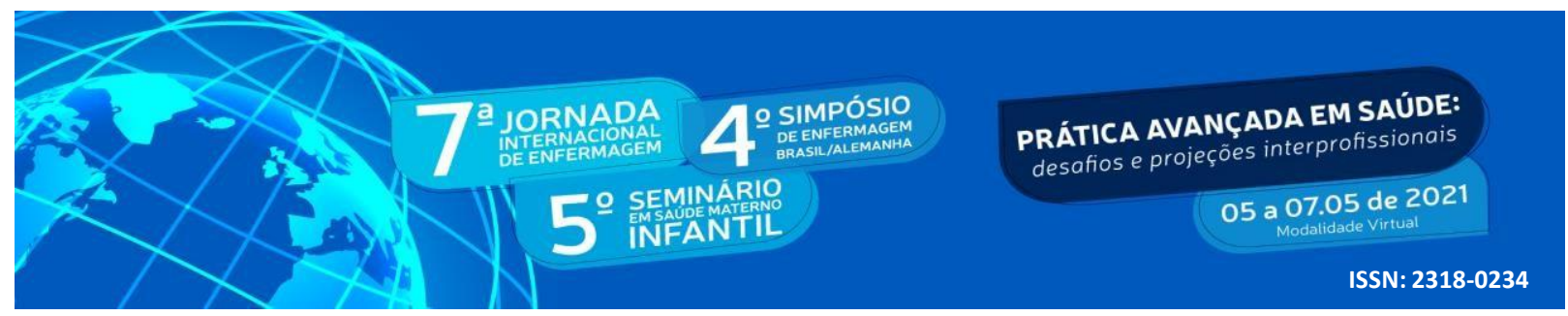

identificar se havia interesse de participar de uma atividade em grupo online, se ela dispunha de acesso a internet e qual temática ela gostaria que fosse abordada. Para esse último, foi utilizada uma pergunta aberta, que não direcionasse a resposta para algum tema e que não fosse realmente uma necessidade do público alvo. Cada tema será abordado em diferentes encontros e será aplicado uma sessão de TBL para cada assunto proposto.

Dentre as temáticas elencadas pelas gestantes prevaleceu: parto; cuidados com RN e amamentação. A fim de facilitar a abordagem e viabilizar a TBL nesse primeiro momento, as facilitadoras elegeram a temática do parto e elencaram as dúvidas frequentes identificadas na prática clínica para servir como disparadores das discussões entre os grupos na tentativa de produzir orientações gerais.

Elegeram-se os seguintes questionamentos referentes à temática do parto para as discussões: 1) Com a proximidade do parto, o que é preciso estar organizado para ser levado à maternidade? 2) Quais são os direitos da gestante na hora do parto? 3) Quais os tipos de parto? 4) Quais as vantagens do parto vaginal para a mãe e o bebê em relação a cesariana? 5) O que você sabe sobre a cesariana? 6) Quais os sinais que indicam o início do trabalho de parto? 7) O que você pode fazer para facilitar o trabalho de parto? 8) Quais as posições que a gestante pode adotar para o trabalho de parto? 09) Quais as providências a serem tomadas após o nascimento do bebê para realizar a certidão de nascimento? 10) Quando devo ir para a maternidade?

Em relação aos resultados prévios destacam-se ainda as etapas da metodologia da TBL que culminaram na elaboração dessa proposta. Assim, em um primeiro momento, as facilitadoras apresentarão a atividade para o grupo de gestantes, informando como será realizada, e também apresentarão os disparadores sobre a temática. A seguir o grupo será dividido em subgrupos. Cada uma das facilitadoras acompanhará um subgrupo, sem interferir nas discussões e sem dar orientações em relação aos temas, para que o conhecimento prévio de cada gestante não sofra a influência dos facilitadores e cada grupo buscará um consenso em cada um dos tópicos discutidos. Depois de realizada as discussões nos pequenos grupos, seguirá a socialização no grande grupo sem que sejam avaliados como correto ou não, elencando-se as dúvidas de cada tópico entre os diferentes grupos. 


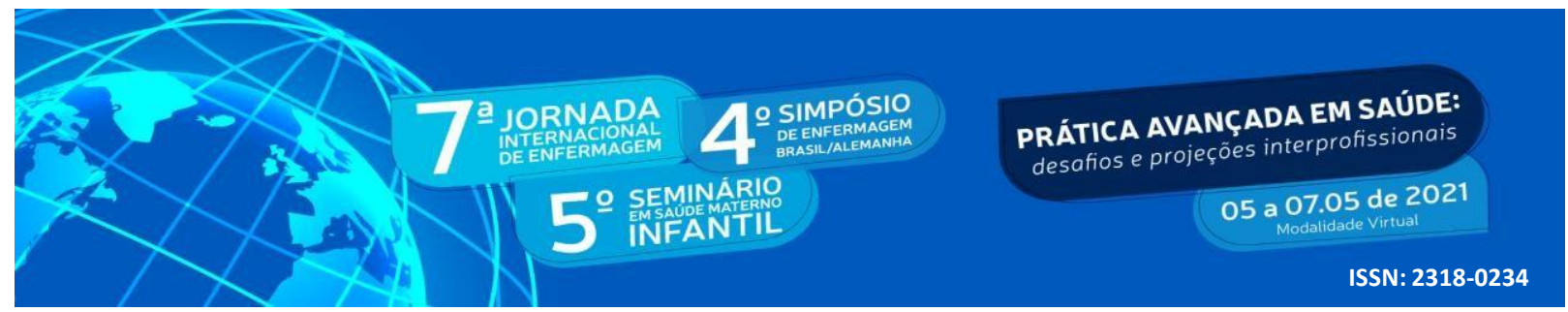

Posteriormente será exibido o vídeo dos especialistas previamente preparados, com as informações sobre a temática parto embasadas cientificamente, com o conhecimento atual sobre cada temática. O subgrupo irá discutir sobre o que foi construído por eles e o que foi exposto pelos especialistas. Cada subgrupo irá apresentar as percepções que tiveram sobre a temática do parto. Os facilitadores auxiliarão na reflexão, identificando os novos conhecimentos adquiridos acerca das discussões durante todo o processo.

\section{DISCUSSÕES PRÉVIAS}

Sabe-se que o conhecimento reproduz atitudes e novos significados para as pessoas (FREIRE, 2014). Para tal, a metodologia TBL envolverá as gestantes a participarem ativamente com as facilitadoras na construção do conhecimento que de acordo com Gonçalves et al (2018) promove maior envolvimento no processo.

A Rede Cegonha (RC), criada em 2011, fomenta a implementação de novos modelos de atenção à saúde da mulher e à saúde da criança priorizando a atenção ao parto, nascimento, crescimento e o desenvolvimento da criança de zero aos vinte e quatro meses (BRASIL, 2017). Assim, as boas práticas de atenção ao parto e nascimento devem ser baseadas em evidências científicas para redução do número expressivo de mortes maternas e neonatais com vistas ao alcance das metas do milênio pactuadas entre os países do mundo.

A Política Nacional de Humanização, criada em 2011, na atenção à saúde da mulher implica a promoção, o reconhecimento e o respeito aos direitos humanos, incluindo os eventos da gestação, parto e nascimento, de forma a colocar a mulher como protagonista no processo de decisão pelo tipo de parto, incluído também o direito a um acompanhante à sua escolha desde a internação até o momento do pós-parto (BRASIL, 2014). No entanto, a prática clínica ainda evidencia uma contrapartida com as propostas dessa política, pois além de uma medida governamental, necessita perpassar pelo comprometimento dos profissionais de saúde envolvidos (VALADÃO; PEGORARO, 2020).

Para tal, além da sensibilização dos profissionais de saúde, necessita-se também atuar frente às atividades educativas em saúde para que as mulheres por meio do conhecimento favoreçam o protagonismo e empoderamento nesse momento de suas vidas (BRAGA et al., 


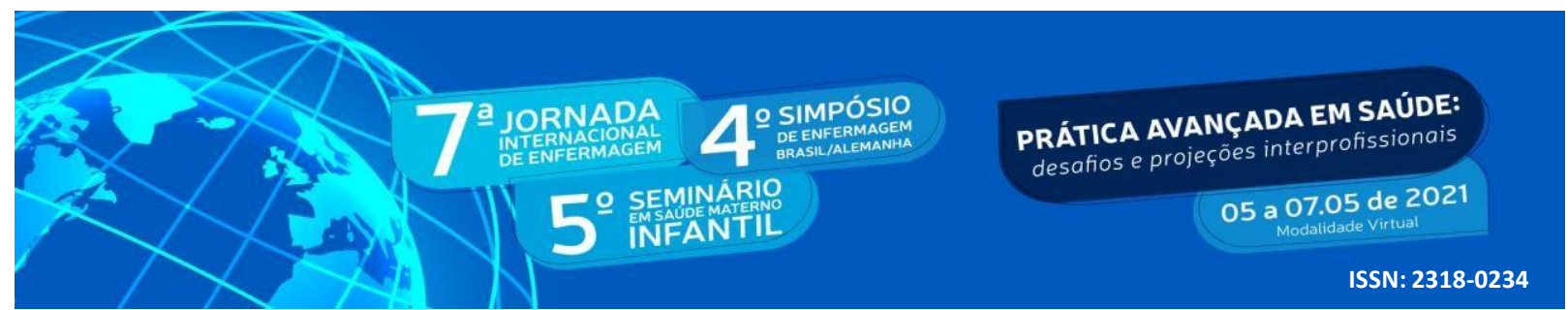

2020). O desconhecimento do ciclo gravídico-puerperal torna a gestante mais suscetível às práticas de hipermedicalização, ao número abusivo de cesarianas e intervenções desnecessárias (SILVA, 2017).

Nessa perspectiva, o cenário de atuação dos profissionais de saúde em atividades de pré-natal torna-se o momento ideal para oportunizar a escuta dessa mulher, troca de experiências grupal, consolidar vínculos com os profissionais da UBS, contribuindo para o incentivando ao parto normal e redução da morbimortalidade materna e infantil (MEDEIROS et al., 2017).

\section{CONSIDERAÇÕES}

Espera-se que a atividade educativa mediada pela metodologia TBL facilite a abordagem das temáticas elencadas pelas gestantes, promovendo assim a aprendizagem significativa, pois se acredita que o grupo de gestantes é uma alternativa que pode possibilitar o acolhimento dessas demandas e espaço para promoção de saúde capaz de ressignificar as experiências vivenciadas.

\section{REFERÊNCIAS}

BRAGA, O.R. et al. Orientação às gestantes acompanhadas no pré-natal por equipes multiprofissionais de saúde da família. Research, Society and Development, v. 9, n. 10, e7929109054, 2020.

BRASIL. Ministério da Saúde. Humanização do parto e nascimento. Brasília: MS, 2014.

BRASIL. Ministério da Saúde. Secretaria de Ciência, Tecnologia e Insumos Estratégicos. Departamento de Gestão e Incorporação de Tecnologias em Saúde. Diretrizes nacionais de assistência ao parto normal: versão resumida [recurso eletrônico] / Ministério da Saúde, Secretaria de Ciência, Tecnologia e Insumos Estratégicos, Departamento de Gestão e Incorporação de Tecnologias em Saúde. - Brasília: Ministério da Saúde, 2017.

FARIAS, P. A. M.; MARTIN, A. A. CRISTO, C.S. Aprendizagem Ativa na Educação em Saúde: Percurso Histórico e Aplicações. Revista Brasileira de Educação Médica. v. 39, n.1, p.143-158, 2015. 


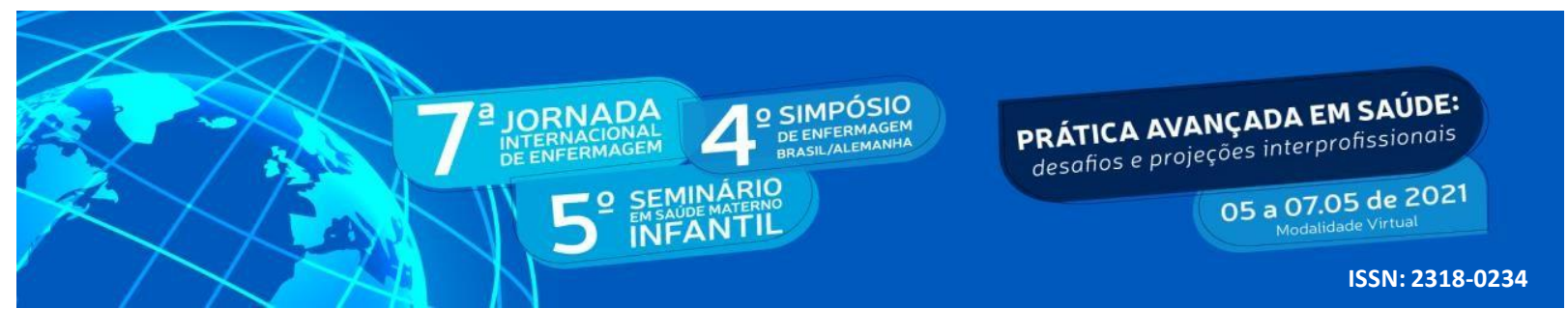

FREIRE, P. Pedagogia da autonomia: saberes necessários à prática da educação. 49. ed. Rio de Janeiro: Paz e Terra, 2014.

GONÇALVES, A. I. M. et al. O uso do team-based learning como metodologia de ensino de saúde coletiva na graduação em odontologia. Revista Brasileira de Educação em Saúde. v. 8, n.1, p.01-06, jan-mar, 2018.

GURGE, C.P.P. TBL no processo de ensino aprendizagem. Periódico Científico Projeção e Docência. v. 6, n.2, 2015.

MEDEIROS, R. M. K. et al. Aspectos relacionados à preferência da gestante pela via de parto. Revista Eletrônica Gestão \& Saúde, v. 1, n.3, p. 603-621, 2017.

OLIVEIRA, B. L. C. A.; LIMA, S.F.; RODRIGUES, L.S. JÚNIOR, G.A.P. Team-Based Learning como Forma de Aprendizagem Colaborativa e Sala de Aula Invertida com Centralidade nos Estudantes no Processo Ensino- Aprendizagem. Revista Brasileira de Educação Médica. v. 42, n.4,p. 86-95, 2018.

PEREIRA, S.B. et al. Boas práticas de atenção ao parto e ao nascimento na perspectiva de profissionais de saúde. Rev Bras Enferm [Internet].v. 71, p.1393-1399, 2018.

ORGANIZAÇÃO DAS NAÇÕES UNIDAS (ONU)- PROGRAMA DAS NAÇÕES UNIDAS PARA O DESENVOLVIMENTO (PNUD). Acompanhando a agenda 2030 para o desenvolvimento sustentável: subsídios iniciais do Sistema Nações Unidos no Brasil sobre a identificação de indicadores nacionais referentes aos objetivos de desenvolvimento sustentável/ Programa das Nações Unidas para o Desenvolvimento. Brasília: PNUD, 2015. Disponível em:

https://www.br.undp.org/content/brazil/pt/home/library/ods/acompanhando-a-agenda2030.html. Acesso em 04 mar.2021.

SILVA, T. C. et al. As boas práticas de atenção ao parto e nascimento sob a ótica de enfermeiros. Biblioteca Lascasas, v.16, n 01, 2016. Disponível em:

http://www.indexf.com/lascasas/documentos/lc0886.php. Acesso em: 04 mar.2021.

SILVA, M. F. Informações sobre parto e puerpério, estratégias e conteúdo da educação em saúde no pré-natal. Dissertação (Mestrado em Ciências) - Instituto Nacional de Saúde da Mulher, da Criança e do Adolescente Fernandes Figueira, Rio de Janeiro, Rio de Janeiro, p.95. 2017.

VALADÃO, C.L. PEGORARO, R.F. Vivências de mulheres sobre o parto. Fractal: Revista de Psicologia, v. 32, n. 1, p. 91-98, 2020. 